\section{Journal ${ }^{\text {of Microbiological }}$ Methods}

\title{
Specific and selective biosensor for Salmonella and its detection in the environment
}

\author{
E.V. Olsen ${ }^{\mathrm{a}}$, S.T. Pathirana ${ }^{\mathrm{b}}$, A.M. Samoylov ${ }^{\mathrm{b}}$, J.M. Barbaree $^{\mathrm{a}}$, \\ B.A. Chin ${ }^{\text {c }}$ W.C. Neely ${ }^{\mathrm{d}}$, V. Vodyanoy ${ }^{\mathrm{b}, *}$ \\ ${ }^{a}$ Department of Biological Sciences, Auburn University, Auburn, AL 36849, USA \\ ${ }^{\mathrm{b}}$ Department of Anatomy, Physiology and Pharmacology, 109 Greene Hall, Auburn University, Auburn, AL 36849, USA \\ ${ }^{\mathrm{c}}$ Material Research and Education Center, Auburn University, Auburn, AL 36849, USA \\ ${ }^{\mathrm{d}}$ Department of Chemistry, Auburn University, Auburn, AL 36849, USA
}

\begin{abstract}
The specific and selective detection of Salmonella typhymurium based on the use of a polyclonal antibody immobilized by the Langmuir-Blodgett method on the surface of a quartz crystal acoustic wave device was demonstrated in liquid samples. These biosensors were selective to S. typhymurium in the presence of large concentrations of Escherichia coli O157:H7. They were also specific to $S$. typhymurium since bacteria preincubated with free antibody produced no signal. Dark-field and electron microscopy showed that two different antibodies, polyvalent somatic $\mathrm{O}$ and flagellar H7, were immobilized on the sensor surface producing two distinct attachments of bacteria at the liquid-solid interface. The somatic $\mathrm{O}$ antibody exhibits a rigid, binding, while the flagellar H7 antibody forms a flexible connection allowing a large degree of freedom. When the attachment of bacteria was rigid and strong, the responses of the acoustic wave sensors correlated with changes in the mass of bacteria present at the liquid-solid interface. In contrast, when attachment was flexible, the sensor signals were inversely proportional to the additional mass of bound bacteria. This difference is probably determined by the interfacial viscoelasticity and by acoustic and electromagnetic coupling. The signals of environmentally aged sensors with either predominately rigid or flexible positioning of bacteria were correlated with changes in mass at the liquid-solid interface. Sensors with $\mathrm{O}$ or $\mathrm{H}$ type of binding could be used for analytical purposes.
\end{abstract}

(C) 2003 Elsevier Science B.V. All rights reserved.

Keywords: Acoustic wave device; Biosensor; Langmuir-Blodgett monolayer; Antibody

\section{Introduction}

The thickness-shear mode (TSM) acoustic wave sensor is proven to be an excellent analytical tool for

\footnotetext{
* Corresponding author. Tel.: +1-334-844-5405; fax: +1-334844-5388.

E-mail address: vodyavi@vetmed.auburn.edu (V. Vodyanoy).
}

the study of specific molecular interactions at the solid-liquid interface (Bunde et al., 1998; O'Sullivan and Guilbault, 1999; Cavicacute et al., 1999; Ivnitski et al., 1999; Kaspar et al., 2000). Acoustic waves in TSM are excited by the application of a radio frequency alternating voltage to the piezoelectric crystal. Changes in the resonance frequency are usually attributed to the effect of the added mass due to the binding at the solid-liquid interface. Acoustic wave 
sensors with immobilized biological recognition molecules (biosensors) were utilized for the real-time study of the adsorption of biochemical macromolecules (Ghafouri and Thompson, 1999). Acoustic wave devices were shown to be quite specific immunosensors in complex biological media containing cells and human serum (Dahint et al., 1999). The theoretical model of the responses of TSM resonators was proven experimentally under various loading conditions, including an ideal mass layer (thin layers of gold and $\mathrm{SiO}_{2}$ ), a semi-infinite fluid (glycerol in water), and a viscoelastic layer exemplified by thin layers of oil (Martin et al., 1991; Bandey et al., 1999). When TSM crystals were exposed to relatively large protein and polysaccharide molecules, the responses obtained for those interactions were not correlated with change in mass imposed at the liquid-solid interface (Ghafouri and Thompson, 1999). The authors ascribed this phenomenon to viscoelastic and acoustic coupling at the interface. One could expect even more complicated interfacial properties when the TSM sensor is exposed to much larger species, like viruses and bacteria. Electromechanical forces created by live and moving organisms may contribute to the apparent mass of the bacteria. Factors such as nutrition, growth, differentiation, chemical signaling, and mutagenic exposure, are important in controlling the bacterial physical state. The bacterial cell (e.g. Escherichia coli), is about 1 million times heavier than a typical $(150 \mathrm{kDa})$ antibody molecule (Neidhardt, 1987). Many bacteria are involved in various movements controlled by flagella, Brownian motion, chemotaxis, swimming behavior, adaptation, and other cell phenomena (Alberts et al., 1989). The ability to bind may also depend on fimbriae and properties of single cells to associate and form colonies. The interaction of bacteria with the biosensor may become dependent on environmental conditions.

The acoustic devices theory and biological applications were recently reviewed (Kaspar et al., 2000; Cavicacute et al., 1999; Ivnitski et al., 1999).

In our previous work, we demonstrated the feasibility of a biosensor based on Langmuir-Blodgett monolayers of an antibody for the rapid and sensitive detection of Salmonella typhimurium in liquid samples (Pathirana et al., 2000). In the present work, we investigated specificity and selectivity of the biosen- sor under environmental conditions and after environmental aging of the biosensors. Additionally, we studied the effects on the biosensor signal of bacterial positioning at the liquid-surface interface.

\section{Materials and methods}

\subsection{Cultures}

S. typhimurium and E. coli $\mathrm{O} 157: \mathrm{H} 7$ cultures from the Auburn University culture collection were used in these experiments. Each culture was confirmed for identity using traditional biochemical, cell morphology, and serologic tests. The cultures were maintained on Trypticase agar (TSA) slants.

\subsection{Growth of cultures and dilutions}

Each culture was streaked for isolation on TSA plates before inoculation of a fresh culture to trypticase soy broth for overnight incubation at $37{ }^{\circ} \mathrm{C}$ in a shaking water bath incubator. The cells were then washed by centrifugation ( $3500 \mathrm{rpm}$ for $10 \mathrm{~min}$ ) of the broth and resuspension in $10 \mathrm{ml}$ of sterile phosphate-buffered saline (PBS) ( $\mathrm{pH}$ 7.0) then repeating centrifugation and resuspension in $2 \mathrm{ml}$ PBS. Aseptic procedures were used throughout the procedure. Serial dilutions were made with PBS. All tubes were shaken before each pipetting to assure mixing before delivery.

\subsection{Colony forming unit (CFU) determinations}

The number of viable cells in each dilution was determined by spread plating $0.1 \mathrm{ml}$ of each dilution onto duplicate plates of TSA, and incubating $48 \mathrm{~h}$ before making a final count of the $\mathrm{CFU} / \mathrm{ml}$ and calculating the average CFU based on dilutions yielding 30-300 colonies/plate. The tubes with diluted cells were immediately placed on ice and delivered to another laboratory for testing with the sensor.

\subsection{Antibodies}

Antibodies used as capture antibodies on the membranes attached to the sensor were obtained from 
Oxoid (Ogdensburg, NY). For S. typhimurium, a polyvalent somatic $\mathrm{O}$ antibody specific for most Salmonella serovars was employed. To capture E. coli O157:H7, polyvalent H7 (flagellar) antibodies were used. In most cases, the same lot of antibody was used throughout. Reactivity was checked against the target bacterium by a slide agglutination test.

\subsection{Procedures for examining the reactivity of antibodies}

Three types of tests were employed to examine the reactivity of antibodies: (1) a dot blot ELISA test using nitrocellulose or nylon filters with antigen fixed and subjected to chromogenic anti-mouse (for monoclonal antibody) or anti-animal-based antibody conjugated with enzyme assay; (2) kit tests for target organisms; and (3) agglutination tests.

\subsection{Monolayer techniques}

\subsubsection{Surface film balance}

Measurements of surface pressure were performed using a Langmuir-Blodgett film balance KSV 2200 LB (KSV-Chemicals, Finland). This fully computerized system contains a Wilhelmy-type surface balance (range $0-100 \mathrm{mN} / \mathrm{m}$; sensitivity $0.05 \mathrm{mN} / \mathrm{m}$ ), a Teflon trough $\left(45 \times 15 \mathrm{~cm}^{2}\right)$, a variable speed motordriven Teflon barrier $(0-200 \mathrm{~mm} / \mathrm{min})$, and a laminar flow hood. The trough was mounted on a $200-\mathrm{kg}$ marble table. Vibration control was provided by interposing rubber shock absorbers, and by mounting the laminar flow hood on a separate bench. Surface pressure was monitored by the use of a sandblasted platinum plate of $4 \mathrm{~cm}$ perimeter. Temperature of the subphase was controlled $\left( \pm 0.1{ }^{\circ} \mathrm{C}\right)$ by water circulation through a quartz tube coil on the bottom of the trough. Temperature was measured by a thermistor located just below the water interface. Surface pressure data were collected during slow, steady-state compression of the monolayers.

\subsubsection{Monolayer formation and deposition}

2.6.2.1. Phospholipid monolayers. Phospholipid solutions were spread on the surface balance as hexane solutions $(1 \mathrm{mg} / \mathrm{ml})$ containing $2 \%$ ethanol (Ito et al., 1989). The subphase used in the experi- ments was a solution containing $55 \mathrm{mM} \mathrm{KCl}, 4 \mathrm{mM}$ $\mathrm{NaCl}, 0.1 \mathrm{mM} \mathrm{CaCl}_{2}, 1 \mathrm{mM} \mathrm{MgCl} 2$ and $2 \mathrm{mM} 3-(N-$ morpholino)-propanesulfonic acid (MOPS) made with deionized double distilled water ( $\mathrm{pH}$ adjusted to 7.4 with $\mathrm{KOH})$.

\subsubsection{Monolayers with immobilized antibo-} dies. The quartz crystals with gold electrodes for the acoustic wave sensor were cleaned by treatment with $50 \%(\mathrm{v} / \mathrm{v}) \mathrm{HNO}_{3}$ and were rinsed in running distilled water until the acid was completely removed. The quartz crystals were then dried and stored until use. The monolayer was formed on the air-liquid interface by allowing the spreading solution to run down an inclined wettable planar surface that is partially submersed into the subphase. One hundred and fifty microliters of the antiserum was spread on the subphase surface by allowing it to flow down a wet glass plate that crossed the interface. The flow rate down the plate was maintained at about 0.1 $\mathrm{ml} / \mathrm{min}$. After spreading, the glass plate was removed, and the monolayer was allowed to equilibrate and stabilize for $10 \mathrm{~min}$ at $19 \pm 0.1{ }^{\circ} \mathrm{C}$. The monolayer was then compressed at a rate of $30 \mathrm{~mm} / \mathrm{min}$ and the vertical film deposition was carried out with a vertical rate of $4.5 \mathrm{~mm} / \mathrm{min}$ and at a constant surface pressure of $23 \mathrm{mN} / \mathrm{m}$. Seven monolayers of the Salmonella antibody film were transferred to the gold surface of the quartz crystals in this manner. Monolayers containing antibodies were transferred at a constant surface pressure onto the round $\left(d=1^{\prime \prime}\right)$ quartz crystals with gold electrodes for acoustic wave device measurements, or onto standard microscope slides (test slides) for visual observations and cell counting.

\subsection{Bacteria binding measurements}

\subsubsection{Acoustic wave device measurements (AWD)}

Measurements were carried out using Maxtek PM740 or TM-400 monitors (Maxtek, Santa Fe Springs, CA) with a frequency resolution of $0.5 \mathrm{~Hz}$ at $5 \mathrm{MHz}$. The devices were capable of working in both single and dual probe modes. Voltage output of the Maxtek device was recorded and analyzed using a standard personal computer, data acquisition card and software. The voltage output from the Maxtek device is directly related to the resonance frequency of the 
quartz crystal sensor. Changes in the resonance frequency of the quartz crystal sensor were used to monitor the binding of bacteria to the sensor surface. The observed changes in the resonance frequency of the quartz crystal sensor during binding of bacteria is hypothesized to be due both to viscoelastic changes of the LB film-bacteria-near surface fluid media and the mass change associated with the binding of bacteria.

\subsubsection{AWD quartz crystal sensors}

AT-cut planar quartz crystals with a $5 \mathrm{MHz}$ nominal oscillating frequency were purchased from Maxtek, Circular gold electrodes were deposited on both sides of the crystal for the electrical connection to the oscillatory circuit.

\subsubsection{Binding measurements}

The Quartz crystal microbalance was calibrated by the deposition of well-characterized stearic acid monolayers. The sensor covered with the antibody film was positioned in the probe arm of the instrument just before delivery of test solutions. Immediately after the recording was started, $1000 \mu \mathrm{l}$ of the control solution was delivered with a pipette to the dry sensor surface and the voltage was recorded for 4-8 min. Then the test solution was carefully removed with a plastic pipette tip. After removal of the control solution a new recording was initiated and $1000 \mu \mathrm{l}$ of solution containing bacteria was added and the same measuring procedure was followed. Temperature of the test solutions was controlled $\left( \pm 0.1{ }^{\circ} \mathrm{C}\right)$. In order to examine the specificity of bacteria binding by antibody immobilized in the sensor membrane, the sensor was exposed to bacteria previously incubated with a solution of free antibody for the optimized contact time. After all the solutions were tested, the sensor crystal was carefully removed and placed in absolute ethanol and subsequently cleaned with concentrated nitric acid. All the equipment used in the experiment was sterilized with ethanol. The data collected were stored and analyzed off line.

\subsection{Dark-field microscopy}

Optical observation and recording of bacteria binding were performed with an Olympus micro- scope fitted with a 100-W mercury lamp illumination source, a polarizer, a Naessens dark-field condenser (COSE, Canada) and a $100 \times$ objective (oil, NA 1.4). The dark-field images (Vodyanoy et al., 1994) were directed to a DEI-470T Optronics CCD Video Camera System (Optronics Engineering, CA). The system provided real-time, direct-view optical images of high resolution. The samples needed no freezing, dehydration, staining, shadowing, marking, or any other manipulation. They were observed in the natural aqueous environment. A direct count of bacteria was used to determine their concentrations in liquid samples. The count of bound bacteria in the presence of a large concentration of motile cells was used to estimate the surface concentration of specific antibody.

\subsection{Environmental aging of sensors}

One hundred and forty-eight Salmonella antibody sensors and 35 test slides were fabricated by transferring seven monolayers of Salmonella antibody serum to each of the quartz crystal substrates and microscope slides using the LB technique. One hundred and forty samples were divided into five sets: a control set, and sets with samples immersed in raw chicken exudate at temperatures $\sim 4,11,23$ and $33{ }^{\circ} \mathrm{C}$ (Olsen, 2000). Each set of crystals was divided into groups of four crystals and one test slide. The control group was tested immediately after production. Each day for 7 days, four sensors and one test slide from other groups were removed from the chicken exudate, rinsed and tested. The sensor was positioned in the holder of the acoustic wave device and the output voltage of the balance was recorded for $8 \mathrm{~min}$ after $1000 \mathrm{ml}$ of the following solutions were applied in sequence: subphase solution, concentrated salmonella suspension diluted 1/625, 1/125, 1/25, 1/5 and undiluted bacterial suspension. Eight initial (not-aged) sensors were also tested as a control for interaction with Salmonella suspended in chicken exudate and in exudate containing no Salmonella. The bacterial count was measured each day and the concentrations were calculated accordingly. The concentration of bacteria ranged from $10^{7}$ to $10^{10}$ cells $/ \mathrm{ml}$. The dose-response experiments were carried out at a temperature of $25{ }^{\circ} \mathrm{C}$. 


\section{Results and discussion}

\subsection{Rigid versus flexible positioning of bacteria onto the surface of acoustic wave sensor}

When the somatic O antibody against Salmonella was employed the firm, whole body, attachment of bacteria was confirmed by electron and dark field microscopy (Figs. 1 and 2A). Electron micrographs show that bacterial binding in this case is characterized by the parallel alignment of cells with visible filaments firmly attaching cells to the surface. The real time dark-field recordings of live bacteria bound to the surface confirm this observation (Fig. 2A). In contrast, H7 flagellar antibodies provide a flexible attachment of cells to the sensor surface allowing a large degree of freedom. The molecular machinery of a bacterium produces the rotation of its flagella (Schuster and Khan, 1994). When a single flagellum is fixed to the surface by an antibody, the molecular machinery of the bacterium generates a rotation of the whole cell. This rotation can be videotaped; representative frames are shown in Fig. 2B. These figures depicts nine consecutive positions of a rotating bacte-

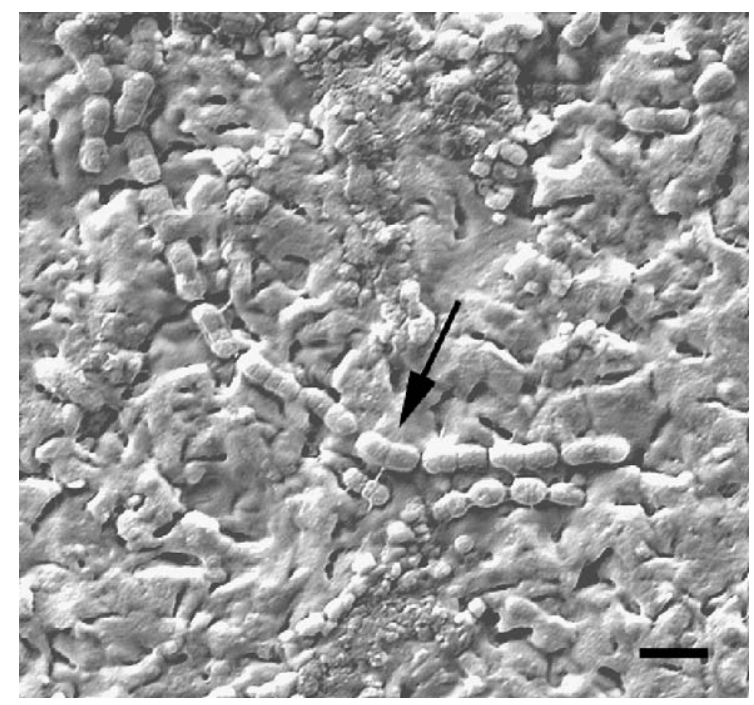

Fig. 1. Scanning electron microscope image of the biosensor surface after exposure to $S$. typhimurium (arrow). The bacteria were attached to the O-type somatic antibody at the rough surface unpolished crystal covered with gold. The image exhibits the rigid attachment of bacteria. Some filaments holding bacteria at the surface are visible. Magnification, $\times 5000 ;$ bar $=5 \mu \mathrm{m}$. rium at intervals of about $100 \mathrm{~ms}$. If several flagella are bound to the surface, the video recording showed side-to-side oscillation of cells (not shown). When sensors with somatic O antibodies against Salmonella were subjected to environmental aging, dark-field observation of the sensors showed an increase in the surface buildup of indigenous bacteria as the time of aging and the temperature were increased (Olsen, 2000). The buildup partially obstructed the antibodies on the sensor surface and the accessibility of antibodies to Salmonella was decreased. Dark-field microscope observation showed that the firm, whole body, binding of Salmonella was replaced with a loose attachment of bacteria with a considerable degree of freedom as the time of the sensor aging and the temperature were increased. Thus, the positioning of Salmonella at the sensor surface changed from rigid to flexible as environmental aging occurred.

\subsection{Validation of mass measurements of monolayers}

The deposition of increasing numbers of stearic acid monolayers onto the surface of an acoustic wave crystal resulted in a linear increase of the mass (Fig. 3). The deposition of a single monolayer of the stearic acid on the crystal adds an additional mass of $2.5 \times 10^{-7} \mathrm{~g} / \mathrm{cm}^{2}$ (for a $38 \mathrm{mNm}^{-1}$ transfer surface pressure). This agrees well with the theoretical estimate based on the molecular area of the stearic monolayer in the condensed state. At this state, the area per molecule is $\sim 20 \AA^{2}$ (for a single alkyl chain) (Davies and Rideal, 1963). The number of the stearic acid molecules in a monolayer of $1 \mathrm{~cm}^{2}$ is equal to $1 \times 10^{16} / 20=5 \times 10^{14}$. The mass of one stearic acid molecule equals $284 \mathrm{~g} / \mathrm{mol} / 6.023 \times 10^{23}$ molecules $/ \mathrm{mol}=4.72 \times 10^{-22} \mathrm{~g}$. The mass of the single monolayer then is $1 \times 5 \times 10^{14} \times 4.72 \times 10^{-22}$ $\mathrm{g}=2.4 \times 10^{-7} \mathrm{~g}$. This compares well with our experimental value of $2.5 \times 10^{-7} \mathrm{~g}$.

\subsection{Specificity and selectivity of bacterial binding}

Response curves obtained by exposing the sensor to buffer solutions containing different concentrations of the bacteria were characterized by fast reaction, the attainment of a steady-state, and very low non-specific binding (Pathirana et al., 2000). In Fig. 4, curve 1, the 

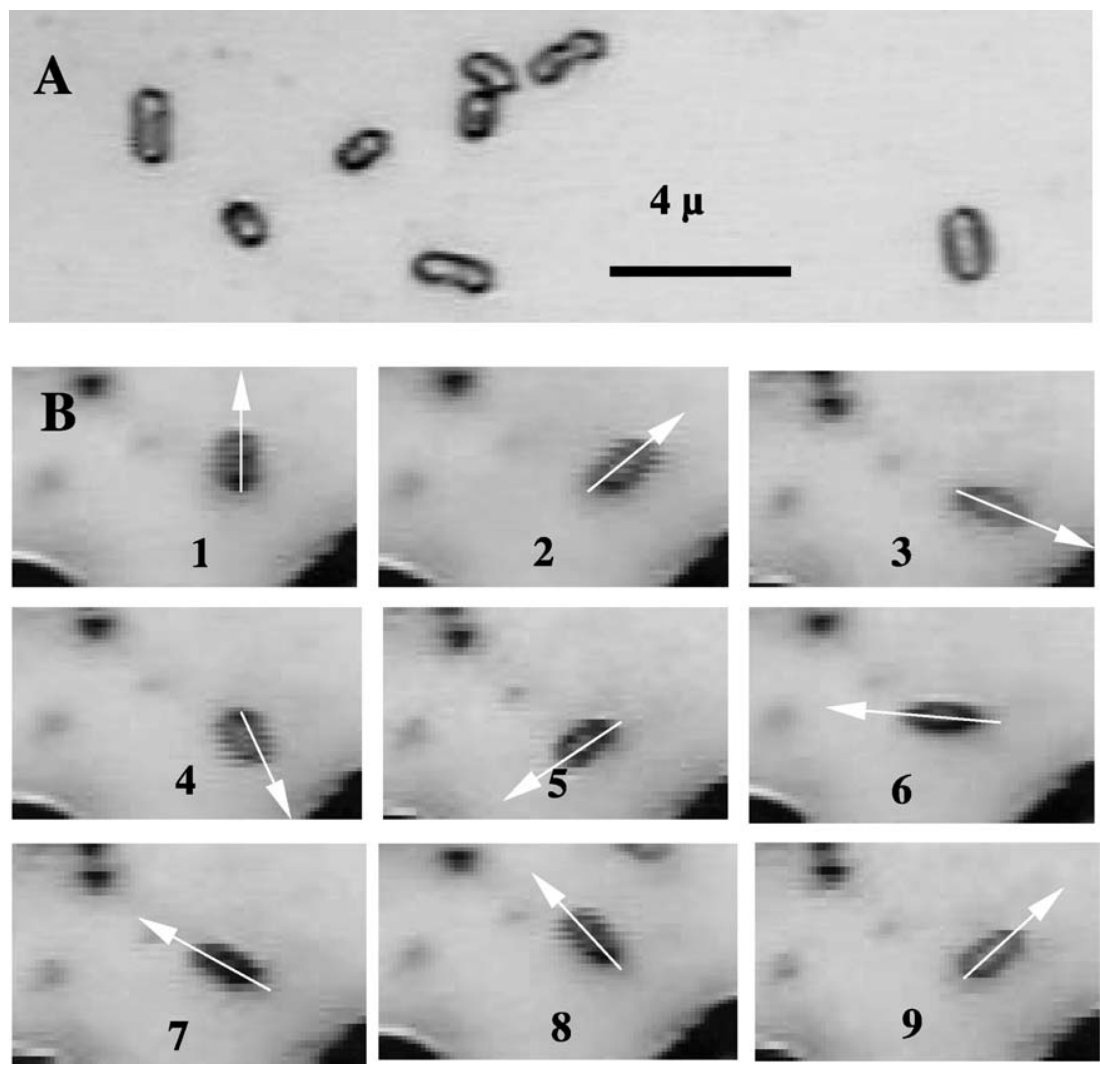

Fig. 2. Dark-field microscope images of bacteria at the surface of the acoustic wave crystal. The images represent time lapse frames from continuous real time video recordings. (A) The rigid attachment of Salmonella to the sensor surface in PBS by somatic O-type antibody. The attached bacteria do not move. (B) The flexible attachment of bacteria with the flagellar H-type antibody allows oscillation and rotation of cells at the crystal surface. Photographs depict rotation of bacterium attached to the antibody by flagellum. Arrows show nine consecutive positions of the rotated bacterium taken at the interval of about $100 \mathrm{~ms}$.

mean values of the steady-state output sensor voltages are plotted as a function of bacteria concentration from $10^{2}$ to $10^{10}$ cells $/ \mathrm{ml}$. The dose response is linear over five decades of bacterial concentration $(R>0.98$, $p<0.001)$. The sensor sensitivity, measured as a slope of the linear portion of the dose response, is $18 \pm 5$ $\mathrm{mV}$ per decade of Salmonella concentration, based on experiments from 112 sensors. The interaction of $S$. typhimurium with the antibody is specific because the sensor does not respond to the bacteria preincubated with the antibody (Fig. 4, line 2). The estimated Hill coefficient (Pathirana et al., 2000), $n$, was found to be equal to $0.45 \pm 0.02$ indicating that two binding sites were needed to anchor one bacterial cell to the sensor surface. Fig. 5 shows the selectivity of the sensor. Line 1 represents the dose response of the Salmonella biosensor to $S$. typhimurium in the presence of $5.6 \times 10^{8}$ cells of $E$. coli. Line 2 show the dose response of the Salmonella sensor to E. coli within the same range of concentration with no Salmonella present. For any given concentration, the sensor response for Salmonella is greater than that for $E$. coli. A marked response difference for Salmonella over $E$. coli is observed even when the number of $E$. coli exceeds the number of Salmonella by a factor of 1000. The non-specific interactions of the sensor with E. coli were small and compare well with those found using an antibody-immobilized QCM (Park et al., 2000; Si et al., 2001; Su et al., 2001; Wong et al., 2002).

The sensors retain $\sim 75 \%$ of their sensitivity (slope of $V$ versus log concentration curve) over a 


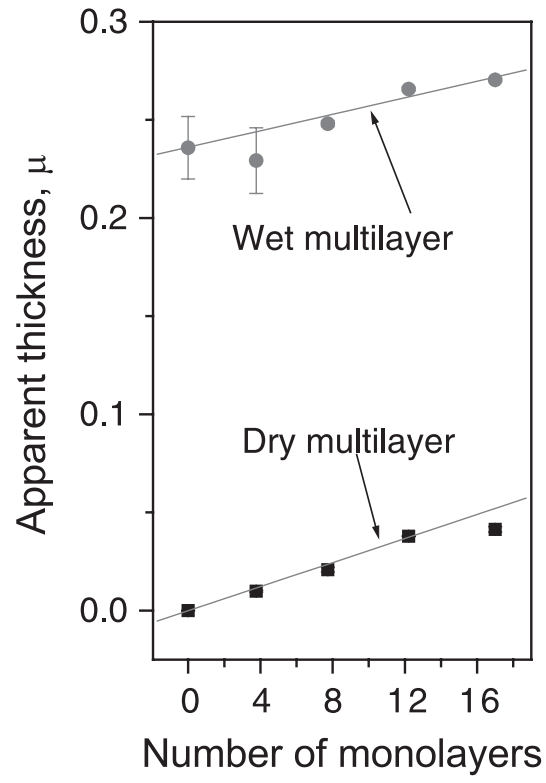

Fig. 3. Validation of measuring mass of bound monolayers by acoustic wave sensor. The experimental thickness of multilayer is a linear function of the number of stearic acid monolayers transferred to the sensor surface. The labels "wet multilayer" and "dry multilayers" indicate sensors submerged in buffer solution and dry sensor, respectively. Points show experimental data \pm SD, while lines represent linear fit $(R=0.921, P<0.02 ; R=0.998, P<0.0001$, respectively).

period of 32 days, and above $25 \%$ after 64 days (Pathirana et al., 2000).

\subsection{Effects of rigid and flexible positioning of bacteria on the apparent mass measured by acoustic wave device}

We found that the bacterial microenvironment and location of the antigen on the surface of a bacterium determines the value and the sign of the analytical signal generated by the acoustic wave device. When molecules of antigen were located on the surface of the bacterial envelope, thus providing firm and tight attachment to the sensor surface, the sensor output voltage was found to be directly proportional to the logarithm concentration of free bacteria in the liquid adjacent to the sensor surface (Fig. 6A, upper line). The lower line of Fig. 6A indicates that bacteria with no antigen matching the sensor antibody, do not bind to the sensor surface. In contrast, when the antibody

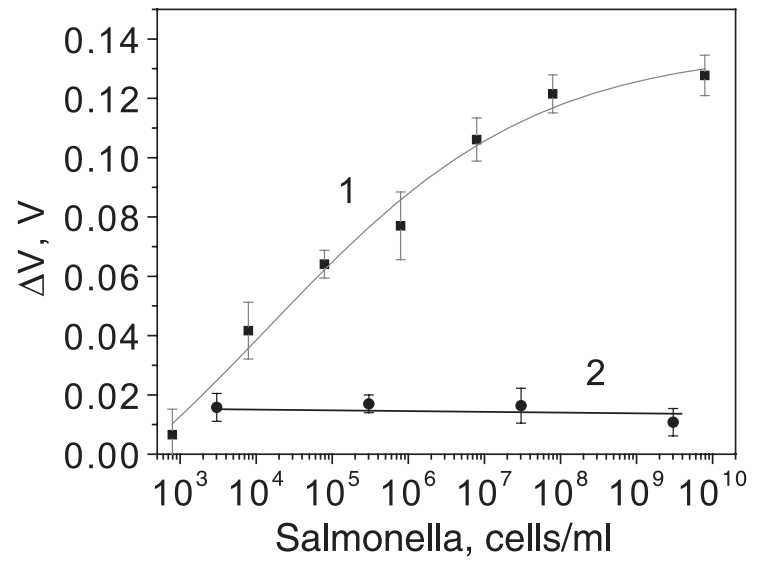

Fig. 4. Specificity of Salmonella sensor. Curve 1 represents the mean values of steady-state sensor voltages as a function of $S$. typhimurium concentrations from $10^{2}$ to $10^{10}$ cells $/ \mathrm{ml}$. The smooth curve is the sigmoid fit to the experimental data $\left(\chi^{2}=5.9 \times 10^{-5}\right)$. Line 2 shows the dose responses of the sensor exposed to $S$. typhimurium suspensions incubated with Salmonella antibodies prior to the exposure. $1.2 \times 10^{9}$ cells were incubated with Salmonella antibodies $(\sim 200 \mu \mathrm{g})$ in $1 \mathrm{ml}$ of PBS for $3 \mathrm{~h} 40$ $\min$. The straight line is the linear least squares fit to the data $\left(R=-0.76\right.$, slope $=-9.0 \times 10^{-5} \mathrm{~V} /$ decade $)$. Experimental data points were obtained by averaging about 200 data points of each steady-state level of response curves; bars are SD.

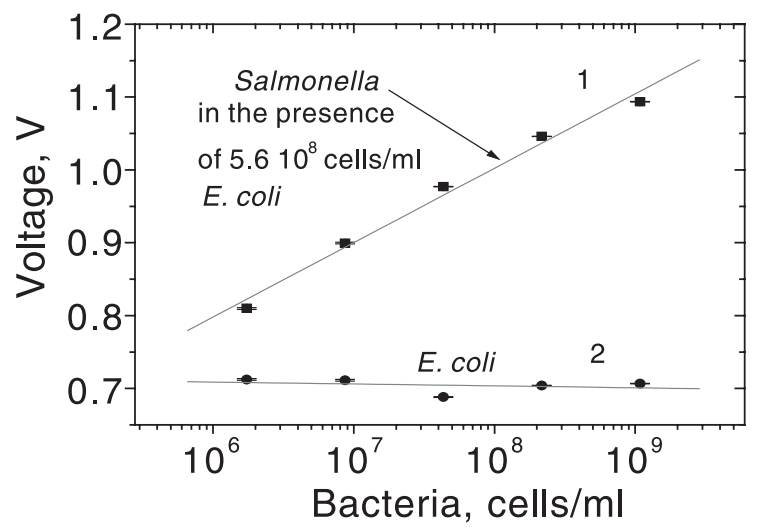

Fig. 5. Selectivity of Salmonella sensor. Curve 1 represents the mean values of steady-state output sensor voltages as a function of S. typhimurium concentrations from $1.8 \times 10^{6}$ to $10^{9}$ cells $/ \mathrm{ml}$ in the presence of $5.6 \times 10^{6}$ cells $/ \mathrm{ml}$ of E. coli $\mathrm{O} 157: \mathrm{H} 7$. The line is the linear fit to the experimental data $(R=0.97)$. Line 2 shows the dose responses of the sensor exposed to E. coli $\mathrm{O} 157: \mathrm{H} 7$. The straight line is the linear least squares fit to the data $(R=-0.71)$. The voltage output was scaled up by a factor of 5 . 

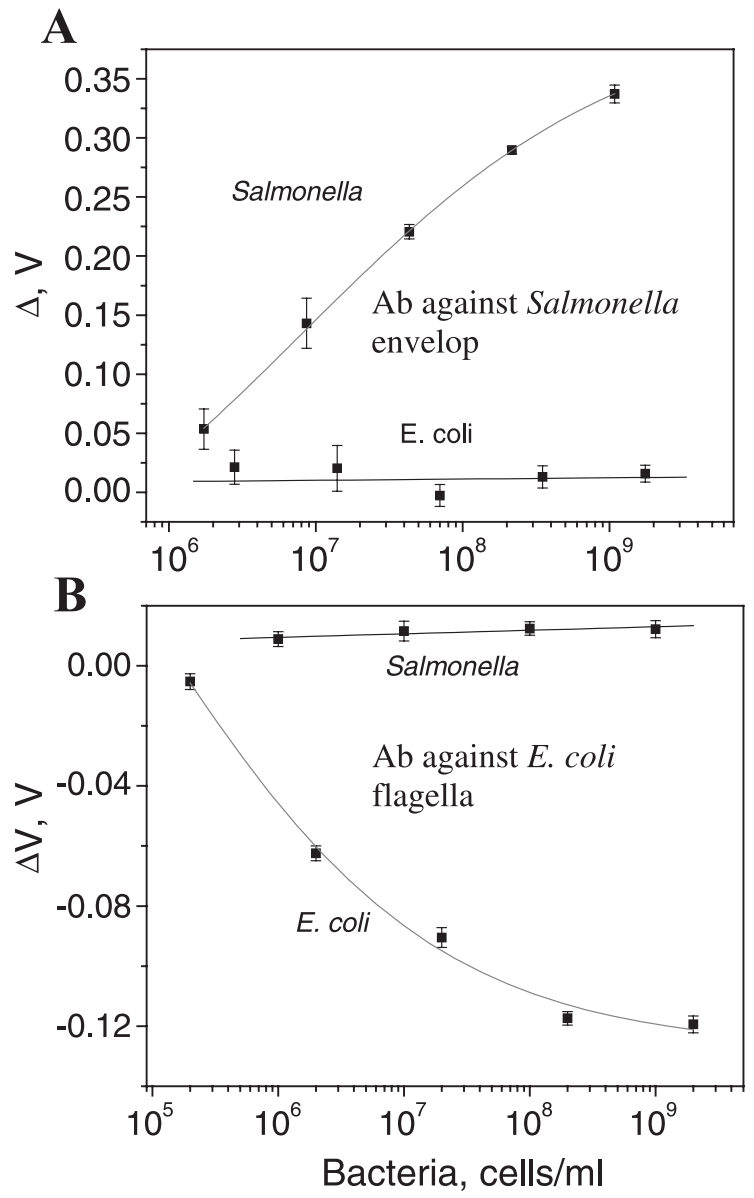

Fig. 6. Dose responses for rigid and flexible positioning of bacteria. (A) The curve labeled "Salmonella" represents Salmonella dose responses of the sensor with the somatic O-type Salmonella antibodies. The line labeled "E. coli" represents E. coli dose responses of the sensor with the somatic O-type Salmonella antibodies. (B) The line labeled "Salmonella" represents Salmonella dose responses of the sensor with the flagellar H-type $E$. coli antibodies. The curve labeled "E. coli" represents $E$. coli dose responses of the sensor with the flagellar H-type $E$. coli antibodies. Curves represent the sigmoid fit to experimental data. The straight lines obtained by the linear least square fit; bars are SD.

was against the antigen located in the bacterial flagellum, the attachment allows a great degree of freedom (rotation and oscillation) so that the output signal of the sensor in was inversely proportional to the logarithm of free bacteria in solution (Fig. 6B, lower line). Again, bacteria with no matching antigen did not bind to the sensor surface (Fig. 6B, upper line). The decrease in voltage output of the sensor corre- sponds to an increase in the resonance frequency of the sensor and hence an "apparent decrease" in effective mass of bacteria attached to the surface. When sensors with somatic $\mathrm{O}$ antibodies against Salmonella were subjected to environmental aging, the type of attachment of bound bacteria was found to depend on the time of exposure. The flexible positioning of bacteria on the sensor surface replaced the rigid one as time increased. With rigid positioning of bacteria, the dose response plots were as shown in Fig. 6A (upper line). When the flexible positioning of bacteria became dominant, and mobility of the bound cells increased, the dose response signal became similar to that of the flagella bound cells shown in Fig. 6B (lower line). Fig. 7 shows that the responses of the somatic antibody type Salmonella sensor aged for 6 days at the temperature $4{ }^{\circ} \mathrm{C}$ decreased as the Salmonella concentration increased. When bacteria were bound with the mixed positioning, the dose response signal depended on the relative contribution of cells with predominantly rigid or predominantly flexible attachment. Control experiments with fresh (not aged) Salmonella sensors measuring Salmonella in the chicken exudate and in exudate alone demonstrated low non-specific adsorption (data not shown).

If we define the sensitivity of the biosensor as the slope of the linear portion of the dose response (signal $\Delta V$ in $\mathrm{mV}$ per decade of Salmonella concentration) then the sensitivity of the sensors is expected to be positive or negative value for the predominantly rigid

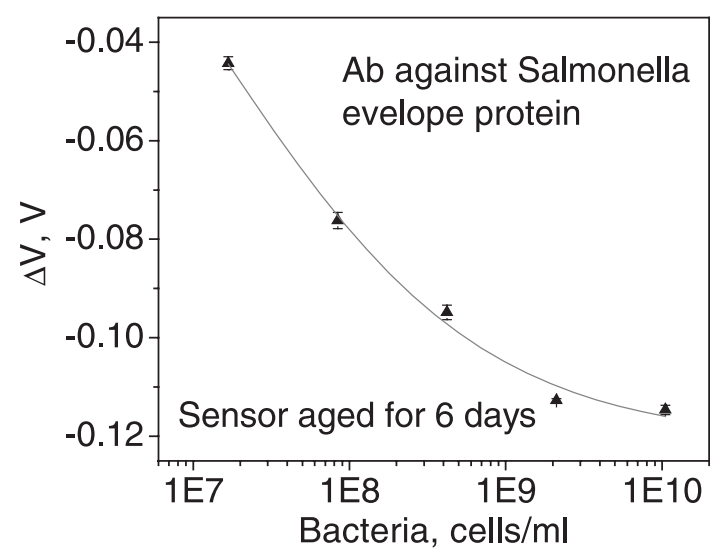

Fig. 7. Dose responses of the sensor with the somatic O-type Salmonella antibodies to Salmonella. The sensor was tested after it was environmentally aged for 6 days at $4{ }^{\circ} \mathrm{C}$. 
or flexible positioning, respectively. The experimental dose response data for environmentally challenged sensors were fitted by a linear regression analysis to a line $\Delta V=A+S \times \log (C)$, the slope of the line (sensitivity), $S$, and the regression coefficient, $R$, were calculated for each sensor. We found the regression coefficient, $R$, ranged from about +0.98 (direct linear correlation), through 0 (no linear correlation) to -0.98 (inverse linear correlation) for positive, zero, and negative sensitivities, respectively (Appendix A). Fig. 8 shows the experimental regression coefficient as the function of sensitivity of Salmonella sensors. The majority of the aged sensor sensitivity values for measurements carried out between 4 and $33{ }^{\circ} \mathrm{C}$ fell between -30 and $+30 \mathrm{mV} /$ decade. It is clear that from these results that the acoustic wave sensor has acceptable analytical value for detecting bacteria only if positioning of bacteria on the sensor surface is either predominantly rigid or predominantly flexible. In the mixed cases the sensitivity and correlation coefficient for dose response signals are not favorable for the effective detection of bacteria. The practical resolution for this phenomenon would be the ensuring of adequate bacterial binding by using antibodies (or

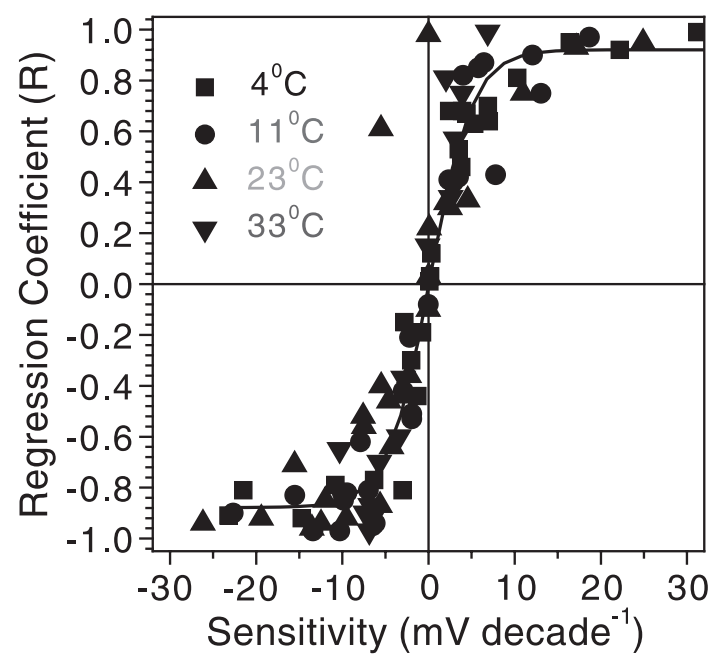

Fig. 8. The experimental regression coefficient as the function of sensitivity of Salmonella sensors with the somatic O-type antibody for environmentally aged sensors. The linear portions of dose response signals were fitted by linear regression. The regression coefficients $(R)$ were plotted against the slopes (sensitivity, $\mathrm{mV} /$ decade) for sensors aged at temperatures indicated in the label. The line is the sigmoid fit, while the points are experimental data. other recognition molecules) with high affinity and multiple binding valences. The antibodies should be specific for either somatic or flagella proteins and they must not to be used simultaneously on the same acoustic wave sensor.

The observed changes of apparent mass as a function of bacterial concentration are hypothesized to be due both to viscoelastic changes of the LB filmbacteria near surface fluid media and the mass change associated with binding of the bacteria. The most peculiar results show that at certain controlled conditions there is an appearance of a negative apparent mass, i.e. with increasing bacterial concentration there can be a dose dependent decrease of the apparent mass. The exact mechanism producing the negative apparent mass is not known. However, this effect may be due to different physical, chemical, and biological mechanisms. These include electrochemical interaction between cells and the diffusion layer of ions on the sensor-liquid interface, high frequency electrophoretic driving force, the viscoelasticity of bacterial cells, physiological effects including electromechanical forces created by the live, moving organisms.

There are two very different positions where bacterial antigens may bind with antibodies immobilized on sensor surfaces: $\mathrm{O}$ somatic antigens, derived from the outer membrane of bacteria, and $\mathrm{H}$ antigens, derived from flagella proteins. Fig. 9 shows these different binding sites and at least three different ways in which the bacterial cells may bind to the sensor surface. Viscoelastic properties of the bacterial layer attached to the surface are anticipated to be different depending on the mechanism of binding: somatic or flagellar. For the three cases shown in Fig. 9, the viscous shear and viscous drag forces of the attached bacteria are very different. It is clear that bacteria at the rigid and flexible positioning (Fig. 9A and B) take different roles in the oscillation of the whole system. When the binding is rigid, bacteria oscillate in unison with the crystal and therefore contribute to the effective oscillating mass of the system. This is shown by the increase of the apparent mass when concentration of bacteria is increased. In the case of flexible attachment, the oscillation of the bacteria may be not in phase with the oscillation of the crystal and thus can cause a decrease in the apparent mass when concentration of the bacteria is increased. Additionally, the electrically charged bacterium on the surface of an 


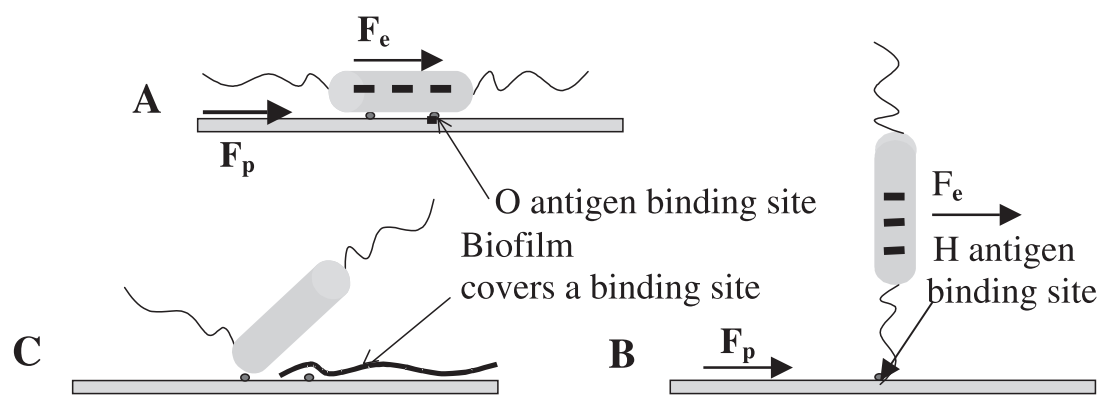

Fig. 9. Different positioning of bacteria at the surface of biosensor. (A) The rigid attachment of bacteria by the somatic O-type antibodies. The bacteria moves in unison with the crystal. The electrophoretic force $\left(F_{\mathrm{e}}\right)$ applied to the electric charges of bacterium ("-") is aligned with the cell body. The piezoelectric force $F_{\mathrm{p}}$ causes the particle displacement at the surface of TSM sensor crystal. (B) The flexible attachment of bacteria by the flagellar H-type antibodies. The bacteria have a high degree of freedom and the displacements of crystal particles may not be in phase with the displacements of the bacteria. (C) In the aged sensors, a biofilm covers certain antibody binding sites and guards against polyvalent attachment of bacteria. As a result, the bound bacteria have more degree of freedom than the bacteria with the firm attachment.

acoustic wave crystal is not only engaged in the mechanical oscillations of the crystal but also directly interacts with the electric field driving the sensor crystal. This field drives the piezoelectric quartz crystal and at the same time creates an electrophoretic force applied to the electrically charged bacteria. The piezoelectric and electrophoretic forces can be of different values and directions, depending on positioning of bacteria by $\mathrm{O}$ antigen (firm positioning, Fig. 9A) or $\mathrm{H}$ antigen (flexible attachment, Fig. 9B) and their combination can contribute to the change of the apparent mass of the bacteria as measured by the acoustic device.

\section{Conclusions}

The results of this work demonstrate the high specificity and selectivity of biosensors based on LB monolayers of an antibody deposited on a piezoelectric crystal for rapid detection of bacteria in liquid samples. A marked selectivity for Salmonella over $E$. coli for Salmonella was observed even when the number of $E$. coli exceeded the number of Salmonella by a factor of 1000 . Results also indicate the importance of positioning of bacteria on the sensor surface. When attachment of bacteria is rigid and strong the responses obtained for the interaction of the bacteria with somatic O-type antibodies on the surfaces of acoustic wave sensors correlated directly with changes in mass imposed at the liquid-solid interface. In contrast, when attachment is flexible, as observed for bacteria attached by flagella to H-type antibodies, the sensor signals were inversely proportional to additional mass, and are probably determined by interfacial viscoelasticity and acoustic and electromagnetic coupling. The responses of environmentally aged sensors with the predominantly rigid or flexible positioning of bacteria were correlated with changes in mass at the liquid-solid interface. Sensors with O or $\mathrm{H}$ type of binding could be used for analytical purposes.

\section{Acknowledgements}

We thank Randy O. Boddie for technical assistance. This work was supported partially by grants from USDA 99-34394-7546 and DARPA MDA97200-1-0011.

\section{Appendix A. Simplified analysis of the thickness-shear mode acoustic resonator}

The acoustic wave resonator (Fig. 10A) admittance $(Y(\omega))$ can be expressed by the equation:

$Y(\omega)=j \omega C_{0}+1 / Z_{\mathrm{m}}$,

where $\omega$ is the angular frequency

$\omega=2 \pi f$, 

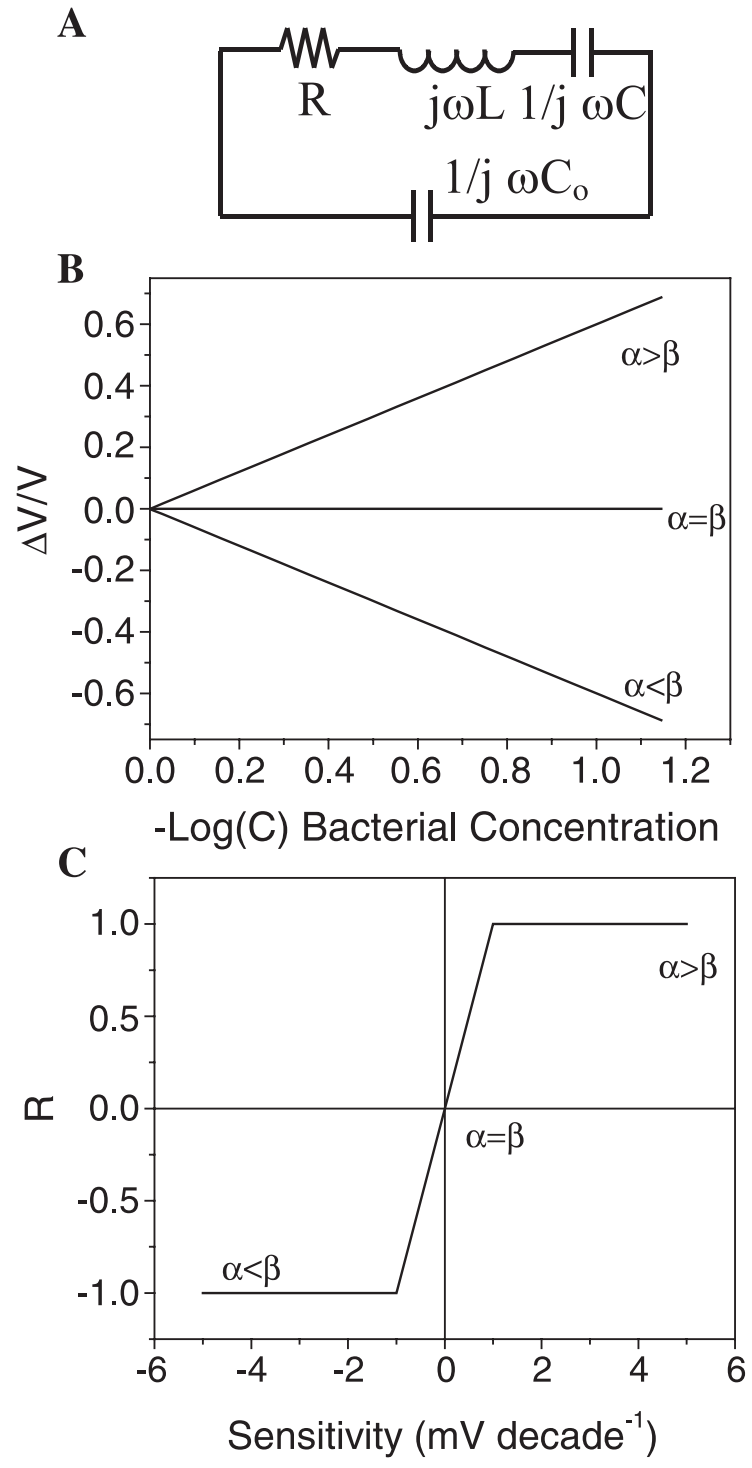

Fig. 10. Simplified electrical model of biosensor. (A) Equivalent circuit of TSM resonator (Martin et al., 1991): a capacitance $C_{0}$ in parallel with resistance $R$, inductance $L$, and functional capacitance C. (B) Dose response dependence described by Eq. (13). (C) Plot of the correlation coefficient as the function of the sensitivity.

$C_{0}$ is the static capacitance of the quartz sensor (we neglect any parasitic capacitance), and $Z_{\mathrm{m}}$ is the motional impedance for the unloaded resonator (Martin et al., 1991).

$Z_{\mathrm{m}}=R+j \omega L+1 / j \omega C$, where $R, L$, and $C$ are the resistance, the inductance and capacitance of the motional arm in parallel with the capacitance $C_{0}$. The series resonant frequency $f$ can be determined as

$f=1 / 2 \pi(L C)^{1 / 2}$.

When the resonator is loaded with solutions the resonant frequency changes due to small changes of parameters $L$ and $C$. The differential of resonant frequency, $\mathrm{d} f$, can be calculated as:

$\mathrm{d} f=(\partial f / \partial L) \mathrm{d} L+(\partial f / \partial C) \mathrm{d} C$,

where partial derivatives $\partial f / \partial L$ and $\partial f / \partial C$ can be calculated from Eq. (4):

$\partial f / \partial L=-(f / 2) / L$

$\partial f / \partial C=-(f / 2) / C$.

Substituting partial derivatives with Eqs. (6) and (7), and replacing the differentials $\mathrm{d} f, \mathrm{~d} L$, and $\mathrm{d} C$ with small changes of the values, we have:

$\Delta f / f=-1 / 2 \times(\Delta L / L+\Delta C / C)$.

Eq. (8) fully agrees with Eq. (27) of Martin et al. (1991) if we consider that $\Delta C / C=0$. If binding bacteria increases the effective mass of the resonator then the serial inductance $(L)$ representing a mass in the equivalent-circuit model, should increase with the increase of bacteria concentration. On the other hand, the binding of the bacteria increases the effective thickness of the resonator and, consequently, decreases the equivalent capacitance $(C)$ of the circuit. The firmly attached dense layer of bacteria creates a small increase of the thickness and the increase in thickness is proportional to the increase in mass. However, bacteria attached by flagella can be separated from the surface as far away as 5-7 $\mu \mathrm{m}$. In this case, the increase of the effective thickness of the bound layer is not proportional to the mass of bacteria because they occupy only a fraction of the layer. The electrically charged layer of bacteria spatially sepa- 
rated from the sensor surface can create a charge distribution, which is equivalent to the serial capacitor connected to the equivalent capacitor of the crystal. We can speculate, further, that relative changes of the inductance and capacitance are linear functions of the bacterial concentration $(C)$ :

$\Delta L / L=\alpha \log (C)$

$\Delta C / C=-\beta \log (C)$,

where coefficients $\alpha$ and $\beta$ are independent of bacteria concentration.

From Eqs. (8)-(10), we have:

$\Delta f / f=-1 / 2 \times \alpha(1-\beta / \alpha) \log (C)$.

The output voltage $(V)$ of the Maxtek PM740 acoustic wave device is inversely related to frequency $f$. So, we can replace $\Delta f / f$ with $-\Delta V / V$ in Eq. (11):

$\Delta V / V=1 / 2 \times \alpha(1-\beta / \alpha) \log (C)$.

Eq. (12) indicates that, if the relative increase of the effective thickness is small compared to increase of the effective mass $(\alpha>\beta)$, then the relative change of the voltage $(\Delta V / V)$ is directly proportional to the logarithm of bacteria concentration (Fig. 10B, line $\alpha>\beta)$. If the opposite is true, and $\alpha<\beta$, then $\Delta V / V$ is inversely related to the concentration of bacteria (Fig. $10 \mathrm{~B}$, line $\alpha<\beta$ ). If $\alpha=\beta$ then $\Delta V / V$ does not depend on the bacteria concentration (Fig. 10B, line $\alpha=\beta$ ):

$\Delta V / V \sim 1 / 2 \times \alpha \log (C), \quad$ if $\alpha>\beta$

$\Delta V / V \approx 0, \quad \alpha \approx \beta ;$

$\Delta V / V \sim-1 / 2 \times \beta \log (C), \quad$ if $\alpha<\beta$.

When experimental dose response data are fitted by a linear regression analysis to a line $\Delta V / V=A+$ $B \log (C)$, the slope of the line (sensitivity), $B$, and the regression coefficient, $R$, are calculated for each sensor. When $\alpha>\beta$, a good positive correlation with a large slope (sensitivity) is also expected to be confirmed by a high regression coefficient $(R \approx 1)$. If $\alpha<\beta$, the regression coefficient at a large negative slope tends to approach a large negative value $(R \approx-1)$. At conditions when $\alpha \approx \beta, R \approx 0$. Thus, the regression coefficient can be a function of the slope and can vary from -1 to +1 , for different sensors with an inverse and direct concentration dependence, respectively (Fig. 10C).

\section{References}

Alberts, B., Bray, D., Lewis, J., Raff, M., Roberts, K., Watson, J.D., 1989. Molecular Biology of Cell. Garland, New York.

Bandey, H.L., Martin, S.J., Cernosek, R.W., Hillman, A.R., 1999. Modeling the responses of thickness-shear mode resonators under various loading conditions. Anal. Chem. 71, 2205-2214.

Bunde, R.L., Jarvi, E.J., Jeffrey, J., Rosentreter, J.J., 1998. Piezoelectric quartz crystal biosensors. Talanta 46, 1223-1236.

Cavicacute, B.A., Hayward, G.L., Thompson, M., 1999. Acoustic waves and the study of biochemical macromolecules and cells at the sensor-liquid interface. Analyst 124, 1405-1420.

Dahint, R., Bender, F., Morhard, F., 1999. Operation of acoustic plate mode immunosensors in complex biological media. Anal. Chem. 71, 3150-3156.

Davies, L.T., Rideal, E.K., 1963. Interfacial Phenomena. Academic Press, New York.

Ghafouri, S., Thompson, M., 1999. Interfacial properties of biotin conjugate-avidin complexes studied by acoustic wave sensor. Langmuir 15, 564-572.

Ito, H., Morton, T.H., Vodyanoy, V., 1989. Small odorant molecules affect steady state properties of monolayers. Thin solid films $180,1-13$.

Ivnitski, D., Abdel-Hamid, I., Atanasov, P., Wilkins, E., 1999. Biosensors for detection of pathogenic bacteria. Biosens. Bioelectron. 14, 599-624.

Kaspar, M., Stadler, H., Weiss, T., Ziegler, C., 2000. Thickness shear mode resonators ("mass-sensitive devices") in bioanalysis. Fresenius' J. Anal. Chem. 366, 602-610.

Martin, S.J., Granstaff, V.E., Frye, G.C., 1991. Characterization of quartz crystal microbalance with simultaneous mass and liquid loading. Anal. Chem. 63, 2272-2281.

Neidhardt, F.C., 1987. Chemical composition of E. coli. In: Neidhardt, F.C. (Ed.), Escherichia coli and Salmonella. American Soc. For Microbiology, Washington, pp. 3-6.

Olsen, E.V., 2000. Functional durability of a quartz crystal microbalance sensor for the rapid detection of Salmonella in liquids from poultry packaging. MS Thesis, Auburn University, Auburn, AL, USA.

O’Sullivan, C.K., Guilbault, G.G., 1999. Commercial quartz crystal microbalances - theory and applications. Biosens. Bioelectron. $14,663-670$. 
Park, I.-S., Ki, W.-Y., Namsoo Kim, N., 2000. Operational characteristics of an antibody-immobilized QCM system detecting Salmonella spp. Biosens. Bioelectron. 15, 167-172.

Pathirana, S.T., Barbaree, J., Chin, B.A., Hartell, M.G., Neely, W.C., Vodyanoy, V., 2000. Rapid and sensitive biosensor for Salmonella. Biosens. Bioelectron. 15, 135-141.

Schuster, C.S., Khan, S., 1994. The bacterial flagellar motor. Annu. Rev. Biophys. Biomol. Struct. 23, 509-539.

Si, S., Li, X., Fung, Y., Zhu, D., 2001. Rapid detection of Salmonella enteritidis by piezoelectric immunosensor. Microchem. J. $68,21-27$.
Su, X., Low, S., Kwang, J., Chew, V.H.T., Li, S.Y., 2001. Piezoelectric quartz crystal based veterinary diagnosis for Salmonella enteritidis infection in chicken and egg. Sens. Actuators, B 75, $29-35$.

Vodyanoy, V., Pathirana, S., Neely, W.C., 1994. Stearic acid assisted complexation of $\mathrm{K}^{+}$by valinomycin in monolayers. Langmuir 10, 1354-1357.

Wong, Y.Y., Ng, S.P., Ng, M.H., Si, S.H., Yao, S.Z., Fung, Y.S., 2002. Immunosensor for the differentiation and detection of Salmonella species based on a quartz crystal microbalance. Biosens. Bioelectron. 17, 676-684. 